\title{
Near-Optimal Sublinear Time Algorithms for Ulam Distance
}

\author{
Alexandr Andoni* Huy L. Nguyen ${ }^{\dagger}$
}

\begin{abstract}
We give near-tight bounds for estimating the edit distance between two non-repetitive strings (Ulam distance) with constant approximation, in sub-linear time. For two strings of length $d$ and at edit distance $R$, our algorithm runs in time $\tilde{O}(d / R+\sqrt{d})$ and outputs a constant approximation to $R$. We also prove a matching lower bound (up to logarithmic terms). Both upper and lower bounds are improvements over previous results from, respectively, [Andoni-IndykKrauthgamer, SODA'09] and [Batu-Ergun-KilianMagen-Raskhodnikova-Rubinfeld-Sami, STOC'03].
\end{abstract}

\section{Introduction}

The edit distance (aka Levenshtein distance) between two strings $A$ and $B$, denoted ed $(A, B)$, is the minimum number of character insertions, deletions, and substitutions needed to transform one string into the other. This distance is of key importance in several fields such as computational biology and text processing, and consequently computational problems involving the edit distance were studied quite extensively.

The Ulam metric is a specialization of edit distance to non-repetitive strings, where a string is non-repetitive if every symbol appears at most once in it. There are several motivations for studying this variant. From a practical perspective, strings with limited or no repetitions appear in several important contexts, such as ranking of objects such as webpages (see, e.g., AJKS02 and Mar95).

From a theoretical point of view, Ulam metric presents a concrete waypoint towards the elusive goal of designing algorithms for edit distance over general (or even binary) strings. Indeed, there are two reasons for this. First, Ulam metric appears to retain one of the core difficulties of the edit distance on general strings, namely the existence of "misalignments" between the

\footnotetext{
${ }^{*}$ Center for Computational Intractability at Princeton University (andoni@mit.edu). The work was done while the author was a student at MIT. Supported in part by NSF CAREER award CCR-0133849, David and Lucille Packard Fellowship and Alfred P. Sloan Fellowship .

${ }^{\dagger}$ Princeton University (hlnguyen@princeton.edu). The work was done while the author was a student at MIT.
}

two strings. In fact, there is no known lower bound that would strictly separate general edit distance from Ulam metric: all known lower bounds are nearly the same (quantitatively) for both metrics. These include non-embeddability into normed spaces results KR06, AK07, lower bounds on sketching complexity AK07, and sub-linear time algorithms $\mathrm{BEK}^{+} 03$. Second, Ulam distance is no harder than edit distance over binary strings, at least up to constant approximation (see Theorem 1.2 from AK07]). Thus, the Ulam metric is a specific roadblock that we must overcome before we may obtain improved results for general edit distance. Moreover, algorithms for Ulam metric have already found applications for a certain smoothed model for edit distance over binary strings AK08. We will discuss this application later.

In this paper, we give a near-tight bounds for estimating the Ulam distance up to a constant approximation, in sublinear time. Formally, given two nonrepetitive strings $A$ and $B$ of length $d$ over an alphabet $\Sigma$, with $|\Sigma| \geq d$, the problem is to output a constant approximation to $R=\operatorname{ed}(A, B)$. We show that $\tilde{\Theta}(d / R+\sqrt{d})$ time is sufficient and required for this problem.

Theorem 1.1. (Upper Bound) There exists a constant $\alpha>1$ for which there exists a randomized algorithm that, given two non-repetitive strings $A, B \in$ $\Sigma^{d}$, approximates $R=\operatorname{ed}(A, B)$ up to factor $\alpha$ in $\tilde{O}(d / R+\sqrt{d})$ time, with $2 / 3$ success probability.

Theorem 1.2. (Lower Bound) For every constant $\alpha>1$, if an algorithm approximates Ulam distance up to a factor $\alpha$ with $\geq 2 / 3$ success probability, then the algorithm must take $\Omega(d / R+\sqrt{d})$ time, where $R$ is the edit distance between the two input strings. The lower bound holds for edit distance over binary strings as well.

Our upper bound improves over the bound of $\tilde{O}(d / \sqrt{R})$ obtained in AIK09. We note that the bound from AIK09 is tight in two extreme regimes: when $R \approx \Theta(d)$ and $R \approx \Theta(1)$. In contrast, our algorithm is tight in all the regimes of $R$, up to logarithmic factors. Our lower bound improves over the bound of $\Omega(d / R+\sqrt{R})$ that follows from $\mathrm{BEK}^{+} 03$ and folklore, giving a tighter (near-optimal) bound when $R=\Omega(\sqrt{d})$. 
We further note that, in comparison, the best known upper bounds for general edit distance are currently much weaker: all sublinear time algorithms achieve a polynomial approximation only. Specifically, $\left[\mathrm{BEK}^{+} 03\right.$ ] can distinguish between ed $(x, y)<d^{1-\epsilon}$ and $\operatorname{ed}(x, y)=\Omega(d)$ in $\tilde{O}\left(d^{1-2 \epsilon}\right)$ time. The algorithm of AO09 can distinguish ed $(x, y)<n^{\alpha}$ from $\operatorname{ed}(x, y)>n^{\beta}$ in $n^{\alpha+2(1-\beta)+o(1)}$ time.

Finally, an application of our upper bound theorem is a near-tight distance estimation algorithm for the smoothed edit distance model over binary strings defined in AK08. There, the authors provided a general reduction from distance estimation in the smoothed model of edit distance over binary strings to distance estimation of (worst-case) Ulam distance. (We will not define precisely the smoothed model of [AK08 as it will not appear further in the present article.)

Corollary 1.1. (Informal) Let $x, y \in\{0,1\}^{d}$ be strings drawn from the smoothed model defined in [AK08]. Then, for every $\epsilon>0$, we can compute $\operatorname{ed}(x, y)$ with $O(1)$ approximation in time $\tilde{O}\left(d^{1+\epsilon} / \operatorname{ed}(x, y)+d^{0.5+\epsilon}\right)$.

1.1 Overview of techniques We now describe the main ideas involved in proving Theorems 1.1 and 1.2 .

Both the upper bound and lower bound exploit the fact that the Ulam metric is decomposable as a sumproduct of Ulam metrics. The sum-product of Ulam metrics is a metric on $k$-tuples of non-repetitive strings, i.e., $\left(A_{1}, \ldots A_{k}\right) \in\left(\Sigma^{\ell}\right)^{k}$, with the distance between tuples $\left(A_{1}, \ldots A_{k}\right)$ and $\left(B_{1}, \ldots B_{k}\right)$ being defined as $\sum_{i=1}^{k} \operatorname{ed}\left(A_{i}, B_{i}\right)$. The resulting metric is a submetric of Ulam (over strings of length $\ell k$ ), as it can be realized by Ulam distance between two strings: $A^{\prime}=A_{1} \circ A_{2} \circ \ldots A_{k}$ and $B^{\prime}=B_{1} \circ B_{2} \circ \ldots B_{k}$, where for each coordinate $i$ we relabel the symbols with new symbols from a fresh alphabet $\Sigma_{i}$, and $\circ$ is the concatenation operator.

Before presenting the ideas behind the upper bound, we rather start by presenting the ideas used for our lower bound, which is both simpler and instructive for presenting the ideas of the upper bound theorem. In the following, we will refer only to the testing problem, which asks to distinguish cases $\operatorname{ed}(A, B)<R$ versus $\operatorname{ed}(A, B)>\alpha R$, for some approximation factor $\alpha$ and fixed threshold $R>1$. We note that considering algorithms for the testing problem is sufficient (and necessary) for both the upper and lower bound theorems.

Lower bound. The main question here is proving the bound of $\Omega(\sqrt{d})$, for $R>\sqrt{d}$, since the bound of $\Omega(d / R)$ follows immediately from the lower bound on testing Hamming distance. We first review the construction from $\left[\mathrm{BEK}^{+} 03\right.$, which gives a weaker bound, of $\Omega(\sqrt{R})$. Suppose the testing algorithm wants to distinguish the case $\operatorname{ed}(A, B)<R$ ("close pair") versus ed $(A, B)>2 R$ ("far pair"). Then, the hard distribution generates $A$ randomly from $\Sigma^{d}$, for $|\Sigma| \gg d$, and $B$ is obtained from $A$ by a cyclic rotation of $A$ by an amount $t$ chosen at random from either $[R]$ (for a "close pair") or $[100 R]$ (for a "far pair"). Then, invoking a birthday paradox argument, one can show that the algorithm must sample $\Omega(\sqrt{R})$ positions in order to distinguish the two distributions.

To prove a sample lower bound of $\Omega(\sqrt{d})$, we consider the sum-product of $k \approx d / R$ copies of Ulam metric on strings of length $R$. To generate a "close pair" $\left(A^{\prime}, B^{\prime}\right)$, we pick $A^{\prime}=\left(A_{1}, \ldots A_{k}\right)$ randomly, and construct $B^{\prime}=\left(B_{1}, \ldots B_{k}\right)$ from $A^{\prime}$ where each $B_{i}$ is a cyclic shift of $A_{i}$ by an amount $t_{i}$ chosen at random from $\left[R^{2} / d\right]$. To generate a "far pair", we do the same, except that for one position $i^{*} \in[d / R]$, we generate $B_{i^{*}}$ from $A_{i^{*}}$ via a cyclic rotation by a random amount $t_{i} \in[R]$. Now, for a coordinate $i$, to distinguish between a random shift $t_{i} \in\left[R^{2} / d\right]$ versus $t_{i} \in[R]$, by a birthday paradox argument, the algorithm needs to sample $\Omega\left(\sqrt{R^{2} / d}\right)$ positions from $A_{i}$ and $B_{i}$. Furthermore, since the algorithm does not know the value $i^{*}$, the algorithm has to sample $\Omega\left(\sqrt{R^{2} / d}\right)$ positions for most of the coordinates $i \in[d / R]$. This gives a total bound of $\Omega(\sqrt{d})$ samples.

Upper bound. We are now ready to describe the ideas behind our upper bound. At a very high level, the upper bound does the converse of the lower bound. Suppose we want to test whether $\operatorname{ed}(A, B)<R$ or $\operatorname{ed}(A, B)>\alpha R$, where the approximation factor $\alpha$ is a large enough constant. First, we decompose the Ulam distance between input strings $A, B$ into sum-product of $k=O(d / R)$ strings of length $\tilde{O}(R)$ by partitioning the strings $A=A_{1} \circ \ldots A_{k}$ and $B=B_{1} \circ \ldots B_{k}$ such that $\operatorname{ed}(A, B)=\sum_{i} \operatorname{ed}\left(A_{i}, B_{i}\right)$. Second, we design an algorithm for distinguishing whether the sum-product of Ulam distances $\sum_{i} \operatorname{ed}\left(A_{i}, B_{i}\right)$ is at most $R$ or is bigger than $\alpha R$. We reduce this step to the problem of gap testing the Ulam distance between two strings: given strings $u, v \in\{0,1\}^{\ell}$, distinguish whether $\operatorname{ed}(u, v)<a$ or $\operatorname{ed}(u, v)>b$ for $a \ll b$. In the third step, we design an algorithm for this gap-testing of Ulam distance, that runs in $\tilde{O}(\ell \cdot \sqrt{a} / b)$ time, where $\ell$ is the length of strings $u, v$. Each of these steps requires additional ideas, which we now briefly sketch.

We implement the first step, of reducing to testing of sum-product of $k=O(d / R)$ Ulam distances of strings of $\tilde{O}(R)$ length, as follows. Note that, in general, we cannot just directly partition $A$ (and $B$ ) into blocks of equal length $d / k$ since, in this case, $\sum_{i} \operatorname{ed}\left(A_{i}, B_{i}\right)$ can become as high as $k \cdot \operatorname{ed}(A, B)$. Instead, we proceed 
as follows. Consider the longest common substring of $A$ and $B$, and let its positions in $A$ and $B$ be $S_{A}$ and $S_{B}$ respectively. We find some matching positions $a_{1}, \ldots a_{k-1} \in S_{A}$ and $b_{1}, \ldots b_{k-1} \in S_{B}$ such that $A\left[a_{i}\right]=B\left[b_{i}\right]$. Then, we partition the strings as $A=A\left[1, a_{1}-1\right] \circ A\left[a_{1}, a_{2}-1\right] \circ \ldots \circ A\left[a_{k-1}, d\right]$ and $B=B\left[1, b_{1}-1\right] \circ B\left[b_{1}, b_{2}-1\right] \circ \ldots \circ B\left[b_{k-1}, d\right]$. We show this can be done such that all the lengths of the substrings are $\tilde{O}(R)$, in $\tilde{O}(d / R+\sqrt{d})$ total time.

In the second step, we reduce testing sum-product of $k$ Ulam metrics, $E=\sum_{i} \operatorname{ed}\left(A_{i}, B_{i}\right)$, to (many invocations of) the gap-testing problem of Ulam distance. The idea is to partition the coordinates $i \in[k]$ into levels corresponding to the contributing weight, and estimate separately the contribution of each level to $E$. Namely, we estimate $c_{j}$, the number of coordinates $i \in[k]$ such that $\operatorname{ed}\left(x_{i}, y_{i}\right) \geq 2^{j}$, for all $j=0, \ldots \log R$. Then, $\sum_{j} c_{j} \cdot 2^{j}$ is a constant-factor approximation to $E$. For each $j$, we estimate $c_{j}$ by subsampling coordinates $i$ with rate $\approx 2^{j} / R$, and, for subsampled $i$ 's, testing whether $\operatorname{ed}\left(x_{i}, y_{i}\right) \geq 2^{j}$. So far, it looks like we did not save much: say, for $j=1$, we subsample most of coordinates $i$ for which we have to test whether $\operatorname{ed}\left(A_{i}, B_{i}\right) \geq R / 2$. Naively, this would take at least $\Omega(\sqrt{R})$ time per coordinate, and $\Omega(d / \sqrt{R})$ for all coordinates. However, we note that, say, a big fraction of coordinates actually have distance $\operatorname{ed}\left(A_{i}, B_{i}\right) \leq O(1)$. Thus, at least for a fraction of $i$ 's, we need to only distinguish between $\operatorname{ed}\left(A_{i}, B_{i}\right) \leq O(1)$ and $\operatorname{ed}\left(A_{i}, B_{i}\right) \geq R / 2$. Indeed, our gap-testing algorithm manages to do so in almost constant time.

More generally, the approach from above requires a gap-tester that can distinguish $\operatorname{ed}(x, y)<a$ versus $\operatorname{ed}(x, y)>b$ for all $a \ll b \leq R$. Our gap tester does so in time $\tilde{O}\left(\ell \cdot \frac{\sqrt{a}}{b}\right)$, where $\ell$ is the length of the strings $x$ and $y$. Note that, for the specific case of $b=O(a)$, our algorithm's performance recovers the performance of the algorithm from AIK09. To obtain our gap-testing algorithm, we develop an alternative characterization of Ulam distance, based on characterizations of ACCL07, GJKK07.

In the end, when using our gap tester in the algorithm for testing sum-product of $k$ Ulam distances of strings of length $\leq \ell$, we obtain a total time of $\tilde{O}\left(\frac{\ell}{R} \cdot(k+\sqrt{k R})\right)$. When $k=O(d / R)$ and $\ell=\tilde{O}(R)$ (as obtained in the first step), the running time becomes $\tilde{O}(d / R+\sqrt{d})$.

We proceed to describing our algorithms and the lower bound in detail.

\section{Preliminaries and Notation}

For a string $A$, let $A[i, j]$ denote the substring of $A$ from position $i$ to position $j$ and, abusing notation, also the set of characters in that substring. If an index $i$ is outside of the string $A \in \Sigma^{l}$, we extend, by convention, the string with extra symbols. Namely, for $i \leq 0$, we let $A[i]=\underline{i}$, and, for $i>l$, we let $A[i]=\overline{(i-l)}$ (in particular the extension is the same for all strings). Then $\Sigma$ will denote the extended alphabet. We assume all $\operatorname{logs}_{\mathrm{s}}$ are in base 2 . In the rest of the paper, we will make extensive use of the Chernoff bounds, which we recall below (see, e.g., MR95]).

FACT 2.1. (Chernoff Bound, MR95) Let $X_{1}$, $X_{2}, \ldots, X_{n}$ be i.i.d. random variables and $p=\mathbb{E}\left[X_{i}\right]$, $\epsilon>0, X_{i} \in\{0,1\}$. Then, we have that

- If $\epsilon \leq 2 e-1$, then $\operatorname{Pr}\left[\left|\sum_{i=1}^{n} X_{i}-p n\right| \geq \epsilon p n\right] \leq$ $2 \cdot e^{-\epsilon^{2} p n / 4}$

- If $\epsilon \geq 2 e-1$, then $\operatorname{Pr}\left[\left|\sum_{i=1}^{n} X_{i}-p n\right| \geq \epsilon p n\right] \leq$ $2^{-(1+\epsilon) p n}$.

\section{Distance Estimation for Ulam Distance}

We now describe our algorithm for sublinear time distance estimation of Ulam distance, thus proving Theorem 1.1 .

The main subroutine is for testing the Ulam distance between two strings. Namely, the tester has the following promise for input strings $A, B \in \Sigma^{d}$, and a given threshold $\log ^{5} d \leq R \leq d$ :

- If $\operatorname{ed}(A, B)<\frac{R}{1400}$, then the tester returns CLOSE with probability at least $2 / 3$.

- If ed $(A, B)>R$ but ed $(A, B) \leq 2 R$, then the tester returns FAR with probability at least $2 / 3$.

We note that such a tester is sufficient to approximate the distance $R^{*}=\operatorname{ed}(A, B)$. Indeed, we can run the tester for Ulam distance for each "guess" $R=d / 2, d / 4, d / 8, \ldots$, and stop once the tester returns "FAR". More precisely, for each guess of $R$, we run the tester for Ulam distance for $O(\log d)$ times and take the majority answer. If the majority answer is "FAR", then we return the current value of $R$ as an approximation to $R^{*}$.

Our tester for Ulam distance is described in Figure 1, and is named UlamTest $(A, B, R)$. The tester works as follows. In step one, we decompose $A$ and $B$ into $k=O(d / R)$ substrings $A_{1}, \ldots, A_{k}$ and $B_{1}, \ldots, B_{k}$ such that the sum $\operatorname{ed}\left(A_{1}, B_{1}\right)+\ldots+\operatorname{ed}\left(A_{k}, B_{k}\right)$ equals ed $(A, B)$. We refer to the distance between $\left(A_{1}, \ldots A_{k}\right)$ and $\left(B_{1}, \ldots B_{k}\right)$ as the sum-product of $k$ copies of Ulam distance. In step two, the algorithm tests whether the sum of Ulam distances ed $\left(A_{1}, B_{1}\right)+\ldots+\operatorname{ed}\left(A_{k}, B_{k}\right)$ is bigger than $R$ or is smaller than $R / 1400$. The first step is described below, and its main statement 
is Lemma 3.1. The second step is described in the next section, Section 4, and its main ingredient is Lemma 4.2 The two lemmas together imply Theorem 1.1.

\begin{tabular}{|l|} 
Procedure UlamTest $(A, B, R)$ \\
1. $a, b, m \leftarrow$ PartialAlign $(A, B, 2 R)$ \\
2. Return UlamProductTest $\left(\left(A\left[1, a_{1}\right], B\left[1, b_{1}\right]\right)\right.$, \\
$\left.\quad \ldots,\left(A\left[a_{d / 2 \beta R}+1, d\right], B\left[b_{d / 2 \beta R}+1, d\right]\right), R\right)$
\end{tabular}

Figure 1: The tester determining whether $\operatorname{ed}(A, B)>R$ or $\operatorname{ed}(A, B)<R / 1400$. We assume that $\operatorname{ed}(A, B) \leq 2 R$. Here, $\beta=C_{1} \log ^{3} d$ for a sufficiently large constant $C_{1}>0$.

$$
\begin{aligned}
& \text { Procedure PartialAlign }(A, B, R) \\
& \text { 1. Split } A \text { and } B \text { into blocks of size } \beta R \text {. } \\
& \text { 2. } m_{0} \leftarrow 0 \\
& \text { 3. For } i \leftarrow 1 \text { to } \frac{d}{\beta R} \\
& \text { 4. For } j \leftarrow 4 \text { to } \log 4 R \\
& \text { 5. Pick a random location } p \text { in }[(i-1) \text {. } \\
& \quad \beta R+4 R, i \cdot \beta R-4 R] \text { of the } i^{\text {th }} \text { block. } \\
& \text { Pick } \gamma \cdot 2^{j / 2} \text { random positions from each } \\
& \text { of } A\left[p, p+2^{j}\right] \text { and } B\left[p+m_{i-1}-2^{j}, p+\right. \\
& \left.m_{i-1}+2 \cdot 2^{j}\right] \text {. } \\
& \text { If there is at least one collision } A[u]= \\
& \quad B[v], \text { then do the following. Choose any } \\
& \quad \text { such collision } u_{i}, v_{i} \text {. Set } m_{i} \leftarrow v_{i}- \\
& \quad u_{i}, a_{i} \leftarrow u_{i}, b_{i} \leftarrow v_{i} \text {. Stop the } j \text { loop } \\
& \text { and jump to the next } i \text {. } \\
& \text { If the } j \text {-loop did not stop, then fail. } \\
& \text { 7. Return vectors } a, b, \text { and } m \text {. }
\end{aligned}
$$

Figure 2: Partial alignment of two strings. Here $\gamma=$ $C_{2} \log d$ for a sufficiently large constant $C_{2}>0$.

As described before, in the first step, we partition strings $A$ and $B$ as $A=A\left[1, a_{1}-1\right] \circ A\left[a_{1}, a_{2}-1\right] \circ$ $\ldots \circ A\left[a_{k-1}, d\right]$ and $B=B\left[1, b_{1}-1\right] \circ B\left[b_{1}, b_{2}-1\right] \circ$ $\ldots \circ B\left[b_{k-1}, d\right]$ for some $k=O(d / R)$, where $a_{i}, b_{i}$ are such that positions $a_{i}, b_{i}$ belong to the longest common subsequence of $A$ and $B$ respectively and $A\left[a_{i}\right]=B\left[b_{i}\right]$. In this case, it is immediate to note that $\operatorname{ed}(A, B)=$ $\operatorname{ed}\left(A_{1}, B_{1}\right)+\ldots+\operatorname{ed}\left(A_{k}, B_{k}\right)$. While this may not always be possible, we show we can do it under the assumption that $\operatorname{ed}(A, B) \leq 2 R$.

To be useful for the second step, we also need that $\left|a_{i+1}-a_{i}\right|,\left|b_{i+1}-b_{i}\right| \leq \tilde{O}(R)$ for all $i$. In the next subsection, we show how to find the positions $a_{i}, b_{i}$ with the required properties.

3.1 Decomposition into a Sum Product of Ulam Distances We now show how to find positions $a_{i}, b_{i}, i \in[k]$, belonging to some fixed longest common subsequence (LCS) of $A$ and $B$ and such that $\left|a_{i+1}-a_{i}\right|,\left|b_{i+1}-b_{i}\right| \leq \tilde{O}(R)$ for all $i$.

The main idea is as follows. Let $S_{A}$ and $S_{B}$ be the positions of the LCS in $A$ and $B$ respectively. First we partition the strings $A$ into substrings of equal length $\beta R$, where $\beta=C_{1} \log ^{3} d$ for large enough constant $C_{1}$. We consider each such substring $A[(i-1) \cdot \beta R+1, i \cdot \beta R]$ and take the corresponding substring of $B$ of length $\beta R$ starting at $s_{i}$ (where the notion "corresponding" will be clear momentarily; for the moment assume that $\left.\left|s_{i}-(i-1) \cdot \beta R\right| \leq O(R)\right)$. For each such pair of substrings $A[(i-1) \cdot \beta R+1, i \cdot \beta R]$ and $B\left[s_{i}, s_{i}+\beta R-1\right]$, we find a pair of positions $a_{i}, b_{i}$ that belong to the sets $S_{A}$ and $S_{B}$. We note that this is always possible since ed $(A, B) \leq 2 R$ and thus for each matching pair of positions $a_{i}, b_{i}$ (in the LCS), we have that $\left|a_{i}-b_{i}\right| \leq 2 R$. The notion of "corresponding substring" roughly means that we sequentially correct the start of the $i^{\text {th }}$ substring of $B$ according to the displacement obtained from the previous matching pair $\left(a_{i-1}, b_{i-1}\right)$; i.e., $s_{i}=(i-1)$. $\beta R+b_{i-1}-a_{i-1}$.

To find one such pair of positions $\left(a_{i}, b_{i}\right)$, we employ random sampling from the two substrings and hope for a collision via the birthday paradox. In general, since the substrings may be at distance up to $O(R)$, we might need to sample roughly $\sqrt{R}$ positions, which proves to be too much (and gives a bound of $\tilde{O}(d / \sqrt{R})$ only).

Instead, the algorithm adapts to the local distance in the $i^{t h}$ pair of substrings of $A$ and $B$. Thus, if the $i^{\text {th }}$ pair of substrings are at distance $f_{i}$, then the algorithm will sample roughly $\sqrt{f_{i}}$ samples for this value of $i$ (since, intuitively, the matching symbols differ in position by at most $f_{i}$, once we make the aforementioned correction to the start of the $B$ 's substring). This adaptation to the local distance between substrings is what gives us the improved bound: indeed, for every sequence $f_{i}$ with $\sum f_{i} \leq O(R)$, we have that $\sum_{i=1}^{d / R}\left(O(1)+\sqrt{f_{i}}\right)=O(d / R+\sqrt{d})$.

The complete details of the algorithm are presented in Figure 2. We prove the following lemma.

Lemma 3.1. (PartialAlign) Consider two nonrepetitive strings $A$ and $B$ at distance $\operatorname{ed}(A, B) \leq R$ for some $R \in[d]$. Let $S_{A}, S_{B}$ be the sets of indices of characters in $A$ and $B$ respectively of the longest common subsequence of $A$ and $B$ (note that $\left.\left|S_{A}\right|=\left|S_{B}\right| \geq d-R\right)$.

Then, with probability at least $2 / 3$, the following all hold. The vectors $a$ and $b$ returned by $\operatorname{PartialAlign}(A, B)$ are subsets of $S_{A}$ and $S_{B}$. Furthermore, $\left|a_{i+1}-a_{i}\right|,\left|b_{i+1}-b_{i}\right| \leq 2 \beta R$ for all $i \in$ $[d / \beta R]$. Finally, the running time of $\operatorname{PartialAlign}(A, B)$ 
is $\tilde{O}(d / R+\sqrt{d})$.

Proof. We prove that all $a_{i}$ are from $S_{A}$ with at least 0.9 probability. Since $A$ and $B$ are non-repetitive and $b_{i}$ are such that $A\left[a_{i}\right]=B\left[b_{i}\right]$, then all $b_{i}$ must be from $S_{B}$ as well.

The proof is by induction on $i$. For convenience of notation, we set $a_{0}=b_{0}=0$. Now assume the inductive hypothesis: that all $a_{k} \in S_{A}$ for $k<i$. We prove that, conditioned on this event, the algorithm generates an $a_{i} \in S_{A}$ with probability at least $1-t_{i}$, where $t_{i}$ is a function of $a_{i-1}$ and will be defined later. We then prove that, conditioned on all $a_{i} \in S_{A}$, we have that $\sum t_{i} \leq O\left(\gamma^{2} \log d / \beta\right)$, which will let us bound the failure probability.

Let $f_{i}$ be the number of "bad positions" from the last match $A\left[a_{i-1}\right]=B\left[b_{i-1}\right]$ to the end of the $i^{\text {th }}$ block in $A$ and $B$. Formally, $f_{i}$ is the number of positions in $A\left[a_{i-1}, i \cdot \beta R\right] \backslash S_{A}$ plus the number of positions in $B\left[b_{i-1}, m_{i-1}+i \cdot \beta R\right] \backslash S_{B}$.

The next claim bounds the probability that a "bad position" $s \notin S_{A}$ appears amongst the collisions for a fixed $j$, where a collision is a sampled pair $(u, v)$ such that $A[u]=B[v]$.

Claim 3.1. Fix some $j \leq \log 4 R$. The probability that a position $s \notin S_{A}$ appears in the set of collisions is at most $\frac{f_{i} \gamma^{2}}{\beta R}$.

Proof. Note that we need to care only about symbols $s \in A[(i-1) \cdot \beta R+4 R, i \cdot \beta R] \backslash S_{A}$. The probability of a fixed such symbol $s$ yielding a collision is bounded by the probability that $s \in A\left[p, p+2^{j}\right]$, times the probability that $s$ is sampled from $A\left[p, p+2^{j}\right]$ and $B\left[p+m_{i-1}, p+m_{i-1}+2^{j}\right]$, which is $\frac{2^{j}+1}{(\beta-8) R} \cdot \frac{\gamma 2^{j / 2}}{2^{j}+1}$. $\frac{\gamma 2^{j / 2}}{3 \cdot 2^{j}} \leq \frac{\gamma^{2}}{\beta R}$. We now apply a union bound over all $s \in A[(i-1) \cdot \beta R+4 R, i \cdot \beta R] \backslash S_{A}$ and use the fact that $\left|A[(i-1) \cdot \beta R+4 R, i \cdot \beta R] \backslash S_{A}\right| \leq f_{i}$, and thus obtain the desired conclusion.

The probability that $a_{i} \notin S_{A}$ is bounded by the probability that, for any $j \leq \log 4 R$, there exists a position $s \notin S_{A}$ that appears amongst the collisions. The latter probability is obtained by applying a union bound over all $j$ to the bound from Claim 3.1, resulting in a bound of $\log 2 R \cdot \frac{f_{i} \gamma^{2}}{\beta R}$.

We also need to bound the probability that a $j$-loop fails to stop. We bound this event using the claim from below, which specifies an upper bound on $j$ at which the $j$-loop will stop.

Before stating and proving the claim, we introduce more notation. Consider fixed $p$ and $j$. Let $T_{j}$ be the set of symbols in $A\left[p, p+2^{j}\right] \backslash S_{A}$, and let $\bar{T}_{j}=$
$A\left[p, p+2^{j}\right] \cap S_{A}$. Then $\mathbb{E}_{p}\left[\left|\bar{T}_{j}\right|\right] \leq \frac{f_{i}}{(\beta-8) R} \cdot 2^{j+1}$. By Markov's inequality, with probability at least $1-\frac{20 f_{i}}{(\beta-8) R}$, we have that $\left|\bar{T}_{j}\right| \leq 0.1 \cdot 2^{j}$.

Claim 3.2. The $j$-loop stops for some $j$ satisfying $2^{j} \leq$ $2 f_{i}$, with probability at least $\frac{21 f_{i}}{(\beta-8) R}$. If $f_{i}=0$, then $j$ loop stops at $j=4$.

Proof. Take the smallest $j$ such that $2^{j} \geq f_{i}$. Condition on the event that $\left|\bar{T}_{j}\right| \leq 0.1 \cdot 2^{j}$. Then $\left|T_{j}\right| \geq 0.9 \cdot 2^{j}$. Note that each $s \in T_{j} \subseteq A\left[p, p+2^{j}\right]$ also appears in $B\left[p+m_{i-1}-2^{j}, p+m_{i-1}+2 \cdot 2^{j}\right]$ by definition of $f_{i} \leq 2^{j}$.

Out of $\gamma 2^{j / 2}$ samples in $A\left[p, p+2^{j}\right]$, at least $0.8 \gamma \cdot 2^{j / 2}$ are in $T_{j}$, with high probability (by usual Chernoff bound for $\gamma=\Omega(\log d))$. Let this set of samples belonging to $T_{j}$ be denoted by $W$. Then, we can compute the probability that, out of the $\gamma 2^{j / 2}$ sampled characters in $B\left[p+m_{i-1}-2^{j}, p+m_{i-1}+2 \cdot 2^{j}\right]$, at least one is also in $W$ : this probability is at least $1-\left(1-\frac{0.8 \gamma 2^{j / 2}}{3 \cdot 2^{j}+1}\right)^{\gamma 2^{j / 2}} \geq 1-e^{-\Omega\left(\gamma^{2}\right)} \geq 1-d^{-\omega(1)}$.

Thus, we have at least one collision and the $j$-loop stops with probability at least $1-d^{-\omega(1)}-\frac{20 f_{i}}{(\beta-8) R} \geq$ $1-\frac{21 f_{i}}{(\beta-8) R}$.

We can now completely bound the probability that $a_{i} \in S_{A}$ and the algorithm finishes successfully the corresponding $i^{t h}$ step. Indeed, this probability is at least $1-\log 2 R \cdot \frac{f_{i} \gamma^{2}}{\beta R}-\frac{21 f_{i}}{(\beta-8) R} \geq 1-\frac{2 \gamma^{2} \log R}{\beta} \cdot \frac{f_{i}}{R}$. We set $t_{i}=\frac{2 \gamma^{2} \log R}{\beta} \cdot \frac{f_{i}}{R}$.

Finally, the probability that there exists some $i$ for which $a_{i} \notin S_{A}$ is at most $\sum_{i} t_{i} \leq \frac{4 \gamma^{2} \log R}{\beta}$. $\frac{\sum_{i} f_{i}}{R}$. We claim that $\sum_{i} f_{i} \leq 4 R$. Indeed, for fixed $i, f_{i}$ is the number of positions in $A\left[a_{i-1}, i \cdot \beta R\right] \backslash S_{A}$ plus the number of positions in $B\left[b_{i-1}, m_{i-1}+i \cdot \beta R\right] \backslash S_{B}$ (conditioned on the fact that $a_{i-1} \in S_{A}$ ). In this case, each position $k \notin S_{A}$ contributes to $f_{i}$ for at most 2 values of $i$ (and same for $k \notin S_{B}$ ). Since also $\left|S_{A}\right|=\left|S_{B}\right| \geq d-R$, we have that $\sum_{i} f_{i} \leq 4 R$. Therefore, the probability that there exists some $i$ for which $a_{i} \notin S_{A}$ is at most $16 \gamma^{2} \log R / \beta<0.1$.

It remains to bound the running time. Assume that all $a_{i} \in S_{A}$. Using Claim 3.2, the running time of the algorithm is $\sum_{i=1}^{d / \beta R} O\left(1+\gamma \sqrt{f_{i}} \log d\right)=$ $\tilde{O}\left(\frac{d}{\beta R}+\gamma \sqrt{\frac{d}{\beta R} \cdot R}\right)=\tilde{O}\left(\sqrt{d}+\frac{d}{R}\right)$, where we have applied the Cauchy-Schwartz the additional $O(\log d)$ appears because of the implementation of checking for collisions).

\section{Tester for Sum-product of Ulam Distance}

In this section, we describe a tester for a sum product of Ulam distances between tuples of strings. Given $k$ pairs 
of strings $\left(A_{1}, B_{1}\right), \ldots,\left(A_{k}, B_{k}\right)$, where each string has length at most $\beta R$, for $\beta>1$, and $\sum_{i=1}^{k} \operatorname{ed}\left(A_{i}, B_{i}\right)=$ $O(R)$, the tester runs in $\tilde{O}(\beta(k+\sqrt{k R}))$ time and has the following promise:

- If $\sum_{i=1}^{k} \operatorname{ed}\left(A_{i}, B_{i}\right)<\frac{R}{1400}$, then the tester returns CLOSE with probability at least $\frac{2}{3}$.

- If $\sum_{i=1}^{k} \operatorname{ed}\left(A_{i}, B_{i}\right)>R$, then the tester returns FAR with probability at least $\frac{2}{3}$.

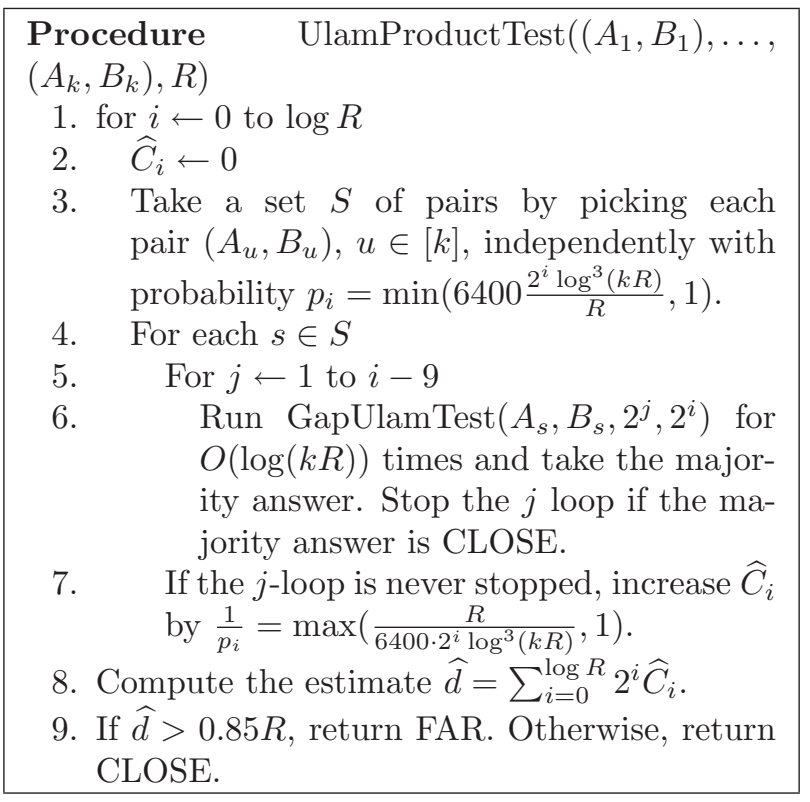

Figure 3: Closeness tester for sum product of Ulam distance.

The tester UlamProductTest is described in details in Figure 3. The idea of the tester is to partition the pairs of strings into buckets of pairs of roughly equal distances and then approximate the number of pairs in each bucket. Specifically, let $C_{i}$ be the number of indices $u$ such that $\operatorname{ed}\left(A_{u}, B_{u}\right) \geq 2^{i}$. For each $i$, we compute an approximation of $C_{i}$ with small additive and multiplicative errors. Finally, an approximation of $\sum_{u=1}^{k} \operatorname{ed}\left(A_{u}, B_{u}\right)$ can be obtained from the sum $\sum_{i=0}^{\log R} 2^{i} C_{i}$. A subroutine GapUlamTest $(A, B, a, b)$ is used to differentiate between the case $\operatorname{ed}\left(A_{u}, B_{u}\right)<a$ and the case $\operatorname{ed}\left(A_{u}, B_{u}\right)>b$. Formally, GapUlamTest satisfies the following properties, which will be proved in a later section.

Lemma 4.1. (GapUlamTest) Suppose we are given two non-repetitive strings $A$ and $B$ of length $\ell_{A}$ and $\ell_{B}$, respectively, of characters in $[d]$ and two constants $a, b$ satisfying $a \leq \frac{b}{512}$. Let $\ell=\max \left(\ell_{A}, \ell_{B}\right)$. With probability at least $2 / 3$, GapUlamTest runs in $\tilde{O}(\ell \sqrt{a} / b)$ time and distinguishes between the case $\operatorname{ed}(A, B)<a$ and the case $\operatorname{ed}(A, B)>b$.

Assuming the properties of GapUlamTest, we now state the formal properties of UlamProductTest.

Lemma 4.2. (UlamProductTest) Given $k$ pairs of nonrepetitive strings $\left(A_{1}, B_{1}\right), \ldots,\left(A_{k}, B_{k}\right)$ of characters in $[d]$ where the length of each string is bounded by $\beta R$ and $\sum_{i=1}^{k} \operatorname{ed}\left(A_{i}, B_{i}\right)=O(R)$. With probability at least $2 / 3$, UlamProductTest runs in $\tilde{O}(\beta(k+\sqrt{k R}))$ time and correctly distinguishes between the cases $\sum_{i} \operatorname{ed}\left(A_{i}, B_{i}\right)>R$ and $\sum_{i} \operatorname{ed}\left(A_{i}, B_{i}\right)<R / 1400$.

Proof. Firstly, we prove the following approximation claim.

Claim 4.1. Consider a set $S$ of n elements and a subset $T$ of $m$ elements. Pick a random subset $X$ of $S$ by picking each element independently with probability $p$. Let $q=|X \cap T|$. Picking $X$ can be implemented in expected $O(p n)$ time and $\operatorname{Pr}\left[|q / p-m| \geq 0.1 m+\frac{6400 \log n}{p}\right] \leq$ $\frac{1}{n^{3}}$.

Proof. We pick $X$ as follows. Divide $S$ into blocks of size $\frac{1}{p}$ and use the binomial distribution to compute the number of samples in each block. Finally, pick the samples from each block according to the computed number of samples. The expected running time is $O(p n)$. Now consider two cases.

1. $m \leq \frac{6400 \log n}{p(2 e-1)}$. By the Chernoff bound, $\operatorname{Pr}[\mid q / p-$ $\left.m \mid \geq m \cdot \frac{6400 \log n}{p m}\right] \leq 2^{-\left(1+\frac{6400 \log n}{m p}\right) p m} \leq \frac{1}{n^{3}}$.

2. $m>\frac{6400 \log n}{p(2 e-1)}$. By the Chernoff bound, $\operatorname{Pr}[\mid q / p-$ $m \mid \geq 0.1 m] \leq 2 e^{-(0.1)^{2} p m / 4} \leq e^{\frac{16 \log n}{2 e-1}} \leq \frac{1}{n^{3}}$.

This concludes the proof of claim 4.1 .

By the Chernoff bound, the probability that the majority answer of $O(\log (k R))$ runs of GapUlamTest (on line 6 of UlamProductTest) is wrong, is bounded by $\frac{1}{k^{3} R^{3}}$. The majority answer is taken $O\left(k \log ^{2}(k R)\right)$ times, so by the union bound, all majority answers from runs of GapUlamTest are correct with probability at least $1-\frac{1}{k R}$. Thus, from now on, we assume all majority answers are correct.

Now we proceed to give upper and lower bounds on $\widehat{C}_{i}$, and hence, the distance estimate $\widehat{d}$. We consider the case $i<\log \frac{R}{6400 \cdot \log ^{3}(k R)}\left(\right.$ so $\left.p_{i}<1\right)$ ). When $i$ is large enough so that $p_{i}=1$, the following bounds still hold because several estimation steps become exact computation. 
We start by showing an upper bound for $\widehat{C}_{i}$ and $\widehat{d}$. Let $N_{i}$ be the number of indices $s \in[k]$ such that $\operatorname{ed}\left(A_{s}, B_{s}\right)>\frac{2^{i}}{512}$. Let $X_{i}$ be the number of indices $s \in S$ such that $\operatorname{ed}\left(A_{s}, B_{s}\right)>\frac{2^{i}}{512}$. By Claim 4.1. $\frac{1}{p_{i}} X_{i} \leq 1.1 N_{i}+\frac{R}{2^{i} \log ^{2}(k R)}$, w.h.p. Hence, $\widehat{C}_{i} \leq \frac{1}{p_{i}} X_{i} \leq$ $1.1 N_{i}+\frac{R}{2^{i} \log ^{2}(k R)}$ and $\widehat{d} \leq \sum_{i} 2^{i}\left(1.1 N_{i}+\frac{R}{2^{i} \log ^{2}(k R)}\right)<$ $1130 \sum_{i=1}^{k} \operatorname{ed}\left(A_{i}, B_{i}\right)+\frac{R}{\log (k R)}$.

Next we show a lower bound for $\widehat{C}_{i}$ and $\widehat{d}$. Let $M_{i}$ be the number of indices $s \in[k]$ such that $\operatorname{ed}\left(A_{s}, B_{s}\right)>2^{i}$. Let $Y_{i}$ be the number of indices $s \in S$ such that $\operatorname{ed}\left(A_{s}, B_{s}\right)>2^{i}$. By Claim 4.1, $\frac{1}{p_{i}} Y_{i} \geq 0.9 M_{i}-$ $\frac{R}{3 \cdot 2^{i} \log ^{2}(k R)}$ w.h.p. Hence, $\widehat{C}_{i} \geq \frac{1}{p_{i}} Y_{i} \geq 0.9 M_{i}-$ $\frac{R}{2^{i} \log ^{2}(k R)}$ and $\widehat{d} \geq \sum_{i} 2^{i}\left(0.9 M_{i}-\frac{R}{2^{i} \log ^{2}(k R)}\right) \geq 0.9$. $\sum_{i=1}^{k} \operatorname{ed}\left(A_{i}, B_{i}\right)-\frac{R}{\log (k R)}$.

Therefore, with probability at least $2 / 3$, if $\sum_{i=1}^{k} \operatorname{ed}\left(A_{i}, B_{i}\right)>R$, then $\widehat{d}>0.9 R-R / \log (k R)>$ $0.85 R$, and if $\sum_{i=1}^{k} \operatorname{ed}\left(A_{i}, B_{i}\right) \leq R / 1400$, then $\widehat{d}<$ $1130 R / 1400+R / \log k R<0.85 R$.

We now prove the stated running time of the algorithm. The expected number of times a fixed pair $\left(A_{u}, B_{u}\right)$ is selected when we compute $\widehat{C}_{i}$ is $O\left(2^{i} \log ^{3}(k R) / R\right)$. When a pair $\left(A_{u}, B_{u}\right)$ is selected, the $j$-loop stops as soon as $2^{j}>\operatorname{ed}\left(A_{u}, B_{u}\right)$. Thus, the expected running time of the algorithm is

$$
\begin{array}{r}
\sum_{i=0}^{\log R} \sum_{u=1}^{k} 2^{i} \log ^{3}(k R) / R \cdot \tilde{O}\left(\frac{\beta R\left(\sqrt{\operatorname{ed}\left(A_{u}, B_{u}\right)}+1\right)}{2^{i}}\right) \\
\leq \sum_{u=1}^{k} \tilde{O}\left(\beta\left(\sqrt{\operatorname{ed}\left(A_{u}, B_{u}\right)}+1\right)\right)=\tilde{O}(\beta(k+\sqrt{k R})) .
\end{array}
$$

4.1 Gap Closeness Tester for Ulam distance In this section, we describe the details of GapUlamTest, a closeness tester differentiating between the case where the Ulam distance is very large and the case where the Ulam distance is very small. Specifically, we have two distance thresholds $a$ and $b$ satisfying $a<b / 512$ and the algorithm should return $F A R$ if the distance is at least $b$, and return CLOSE if the distance is at most $a$.

The tester GapUlamTest is described in details in Figure 4. The idea of the algorithm is to divide both strings into small blocks and estimate the contribution of each block to the total distance. The contribution from each block comes from two sources: character movements within each block, and character movements between different blocks. Intuitively, the first kind of movements can be detected by character inversions within corresponding blocks in two strings. We approximate the number of movements of this kind by the number of characters witnessing a lot of inversions in their neighborhoods (similar to the characterizations from ACCL07, GJKK07, AIK09). The number of movements of the second kind is exactly the difference between the set of characters in the block in the first string and the set of characters in the corresponding block in the second string, which can be approximated by counting collisions between samples from two strings. Furthermore, since only an approximation of the sum of contributions from the blocks is needed, instead of computing the contributions from all blocks, we only sample some subset of blocks to estimate the sum. Specifically, for each $i$, we estimate $n_{i}$, the number of blocks contributing approximately $2^{i}$ or more. The total distance can be estimated by considering the sum $\sum_{i} n_{i} 2^{i}$. Intuitively, the larger $i$ is, the finer the estimation of $n_{i}$ we need, so the number of sampled blocks grows with $i$. On the other hand, the larger the distance $2^{i}$, the easier it is to find mismatches between the corresponding blocks in two strings. It turns out these two effects cancel each other out and for each $i$, we can estimate the contributions from blocks contributing $2^{i}$ or more in $\tilde{O}\left(\frac{\ell \sqrt{a}}{b}\right)$. Summing over all $i$, the total running time is $\tilde{O}\left(\frac{\ell \sqrt{a}}{b}\right)$.

The algorithm uses the following characterization of the Ulam distance in order to approximate the two aforementioned forms of movement. We note that this lemma can be seen as a refinement of the characterizations from [ACCL07, GJKK07, AIK09.

Lemma 4.3. Consider two non-repetitive strings $A$ and $B$. Let $\ell_{A}, \ell_{B}$ be the length of $A$ and $B$, respectively. W.l.o.g. assume $\ell_{A} \leq \ell_{B}$. Define $X=\sum_{k=0}^{\ell_{A} / a} \mid A[k a+$ $1,(k+1) a] \backslash B[(k-1) a+1,(k+2) a] \mid$ i.e. the number of characters occurring in $A[k a+1,(k+1) a]$ but not in $B[(k-1) a+1,(k+2) a]$ for $k \in\left[\ell_{A} / a\right]$. Let $\delta \leq 1 / 2$ be a constant. Define $Y_{\delta}$ to be the number of pairs of indices $u, v$ such that $A[u]=B[v], A[u] \in A[k a+1,(k+1) a] \cap$ $B[(k-1) a+1,(k+2) a]$ for some $k$ and the symmetric difference $|A[u-t, u-1] \Delta B[v-t, v-1]|>2 \delta t$ for some $t \leq 4 a$. Then

- If $\operatorname{ed}(A, B) \leq a$, then $X \leq a$ and $Y_{\delta} \leq 4 a / \delta$.

- If $\operatorname{ed}(A, B) \geq b+\ell_{B}-\ell_{A}$, then $X+Y_{\delta} \geq \frac{b(1-\delta)}{2}$.

Proof. A character is called red if it contributes to either $X$ or $Y_{\delta}$.

First, we show that if $\operatorname{ed}(A, B) \leq a$, then $X \leq a$ and $Y_{\delta} \leq 4 a / \delta$. Let $S_{A}, S_{B}$ be the set of indices of characters in $A$ and $B$ belonging to the longest common subsequence of $A$ and $B$. Note that $\left|S_{A}\right|=\left|S_{B}\right| \geq$ $\ell_{B}-a$. The characters in $S_{A}$ cannot contribute to $X$, so $X \leq a$. Let $T_{\delta}$ be the set of all pairs of indices $u, v$ such that $A[u]=B[v]$, and the symmetric 
Procedure GapUlamTest $(A, B, a, b)$

1. Let $\ell_{A}, \ell_{B}$ be the length of $A$ and $B$. If $\left|\ell_{B}-\ell_{A}\right|>b / 10$, return FAR.

2. Split $A$ into $\ell_{A} / a$ blocks of size $a$.

3. For $i \leftarrow 0$ to $\log a-1$

4. Set $\widehat{X}_{i} \leftarrow 0$

5. Pick a set $S$ of blocks by picking each block $k \in\left[\ell_{A} / a\right]$ independently with probability $\min \left(O\left(\frac{2^{i} \log ^{3} \ell}{b}\right), 1\right)$.

6. For each sampled block $k \in S$

7. If $2^{i} \leq 6400 \sqrt{a} \log \ell$,

8. Compute the number of characters in $A[k a+1,(k+1) a]$ that are not also contained in $B[(k-1) a+1,(k+2) a]$ (i.e., characters contributing to $X$ ) by reading the blocks entirely. Increase $\widehat{X}_{i}$ by 1 if this number of characters is at least $2^{i}$.

9. If $2^{i}>6400 \sqrt{a} \log \ell$,

10. Read each character in $A[k a+1,(k+$ 1)a] and $B[(k-1) a+1,(k+2) a]$ independently with probability $p=$ $\min \left(O\left(\frac{\sqrt{a} \log \ell}{2^{i}}\right), 1\right)$ and let $C$ be the number of collisions between the characters being read in $A$ and in $B$. If $a-\frac{C}{p^{2}}>0.9 \cdot 2^{i}$ then increase $\widehat{X}_{i}$ by 1 .

11. Read each character in $A[k a+1,(k+$ $1) a]$ and $B[(k-1) a+1,(k+2) a]$ independently with probability $r=$ $\min \left(O\left(\frac{\log \ell}{2^{i / 2}}\right), 1\right)$ and find the collisions. For each collision $A[u]=B[v]$, run YContributingTest $(A, B, u, v, a)$. Let $D$ be the number of characters for which the answer is CONTRIBUTING. If $\frac{D}{p^{2}}>$ $0.9 \cdot 2^{i}$, then increase $\widehat{Y}_{i}$ by 1 .

12. Set $\widehat{X} \leftarrow \sum_{i} \frac{b}{\log ^{3} \ell} \widehat{X}_{i}$ and $\widehat{Y} \leftarrow \sum_{i} \frac{b}{\log ^{3} \ell} \widehat{Y}_{i}$

13. If $\widehat{X}+\widehat{Y}>\frac{b}{10}$, return FAR. Otherwise return CLOSE.

Figure 4: Tester distinguishing between the case $\operatorname{ed}(A, B)<a$ and the case ed $(A, B)>b$.

difference $|A[u-t, u-1] \Delta B[v-t, v-1]|>2 \delta t$ for some $t \leq \ell_{B}$. Notice that $Y_{\delta} \leq\left|T_{\delta}\right|$. By [AIK09, Lemma 2.2], $\left|T_{\delta}\right| \leq 4 a / \delta$.

Second, we show the contra-positive of the second assertion, i.e. if $X+Y_{\delta}<\frac{b(1-\delta)}{4}$ then $\operatorname{ed}(A, B)<$ $b+\ell_{B}-\ell_{A}$. We select a common subsequence of $A$ and $B$ by the following removal procedure. For convenience, add two different special characters at the end of $A$ and $B$ and they are not red. Start from the end of $A$ and go back until reaching the beginning of $A$. At position $i$, do the following. If $A[i-1]$ is not red, proceed to $i-1$. If $A[i-1]$ is red, let $j<i$ be the largest index where $A[j]$ is not red and $A[j]$ precedes $A[i]$ in $B$. Remove $A[j+1, i-1]$ and proceed to position $j$. The remaining string after the above process finishes is the common subsequence we need.

Now we bound the number of removed characters when we reach the $i$ th position and $A[i]$ is not red. Because $A[i]$ is not red, $A[i]=B[u]$ for some $u$ satisfying $|i-u| \leq 2 a$. Consider two cases.

1. $i-j \leq 4 a$. Because $A[i]$ is not red, $\mid A[j+1, i-$ 1] $\Delta B[u-i+j+1, u-1] \mid \leq 2 \delta(i-j-1)$. All non-red characters in $A[j+1, i-1]$ contribute to the symmetric difference $\mid A[j+1, i-1] \Delta B[u-i+$ $j+1, u-1] \mid$ so at least $1-\delta$ fraction of the deleted characters are red.

2. $i-j>4 a$. Because $A[i]$ is not red, $\mid A[i-4 a+$ $1, i-1] \Delta B[u-4 a+1, u-1] \leq 8 \delta a$. Thus, in $A[i-4 a+1, i-1]$, at most $4 \delta a$ characters are not red. We now show that all characters in $A[j+1, i-4 a]$ are red. Indeed, any non-red character in $A[j+1, i-4 a]$ must appear after the character $A[i]=B[u]$ in $B$ by the definition of $j$, and thus it contributes to $X$ and is red. Therefore, all characters in $A[j+1, i-4 a]$ are red. In total, at least $4 a-4 \delta a+(i-4 a-j)>(i-j-1)(1-\delta)$ red characters are removed.

In all cases, at least $1-\delta$ fraction of the deleted characters are red. Therefore, we get a common subsequence of $A$ and $B$ of length greater than $\ell_{A}-\frac{b}{2}$ so $\operatorname{ed}(A, B)<b+\ell_{B}-\ell_{A}$. This concludes the proof of Lemma 4.3 .

The tester works by estimating the quantity $X$ and $Y=Y_{\delta}$, for $\delta=1 / 2$ in the above lemma. To detect characters contributing to $Y_{\delta}$, we use the YContributingTest described in Figure 5, The following lemma shows that YContributingTest correctly tests if a character contributes to $Y$.

LEMma 4.4. (YContributingTest) With probability at least $1-\frac{1}{\ell^{2}}$, if $|A[u-z, u-1] \Delta B[v-z, v-1]|>$ $z$ for some $z<4 a$, then YContributingTest returns CONTRIBUTING, and if $\mid A[u-z, u-1] \Delta B[v-z, v-$ $1] \mid<0.8 z$ for all $z<4 a$, then YContributingTest returns NOT-CONTRIBUTING. The expected running time of YContributingTest is $\tilde{O}(\sqrt{a})$.

Proof. Let $N_{t}=\left|A\left[u-1.01^{t}, u-1\right] \cap B\left[v-1.01^{t}, v-1\right]\right|$. Let $D_{t}$ be the number of collisions between samples from $\mid A\left[u-1.01^{t}, u-1\right]$ and $B\left[v-1.01^{t}, v-1\right] \mid$. We have 
$\mathbb{E}\left[D_{t} / q^{2}\right]=N_{t}$ and $\operatorname{Var}\left[D_{t} / q^{2}\right]=\left(1-q^{2}\right) N_{t} / q^{2}$. By the Chebyshev inequality, $\operatorname{Pr}\left[\left|D_{t} / q^{2}-N_{t}\right| \geq 0.05 \cdot 1.01^{t}\right] \leq$ $\frac{400\left(1-q^{2}\right) N_{t}}{q^{2} 1.01^{2 t}}<\frac{1}{10}$. Consider two cases.

1. $|A[u-z, u-1] \Delta B[v-z, v-1]|>z$ for some $z<4 a$. Choose $t=\left\lfloor\log _{1.01} z\right\rfloor$. Then, $\mid A\left[u-1.01^{t}, u-\right.$ $1] \Delta B\left[v-1.01^{t}, v-1\right] \mid>0.98 \cdot 1.1^{t}$. Therefore, $N<0.51 \cdot 1.1^{t}$. By the Chernoff bound, with probability at least $1-\frac{1}{\ell^{2}}$, for the majority of the times, the number of collisions is at most $0.55 \cdot 1.01^{t}$ and the algorithm returns CONTRIBUTING.

2. $|A[u-z, u-1] \Delta B[v-z, v-1]|<0.8 z$ for all $z<4 a$. Therefore, $N>0.6 \cdot 1.01^{t}$. By the Chernoff bound, with probability at least $1-\frac{1}{\ell^{2}}$, the number of collisions is at least $0.55 \cdot 1.01^{t}$ the majority of the times for all $t$ and the algorithm returns NOTCONTRIBUTING.

The expected number of characters being read by YContributingTest, and hence, the expected running time, is $\tilde{O}(\sqrt{a})$. This concludes the proof of Lemma 4.4

Procedure YContributingTest $(A, B, u, v, a)$

1. For $t \leftarrow 0$ to $\log _{1.1} 4 a$

2. Repeat the following $O(\log \ell)$ times.

3. Read each character in $A\left[u-1.01^{t}, u-1\right]$ and $B\left[v-1.01^{t}, v-1\right]$ independently with probability $q=O\left(\frac{1}{\sqrt{1.01^{t}}}\right)$ and count the number of collisions.

4. If the number of collisions is at most 0.55 . $1.01^{t}$ the majority of the times then return CONTRIBUTING.

5. If CONTRIBUTING is never returned then return NOT-CONTRIBUTING.

Figure 5: A procedure for checking if $\mid A[u-t, u-$ 1] $\Delta B[v-t, v-1] \mid$ is large for some $t<4 a$.

Now we proceed to proving Lemma 4.1 .

Proof. We first show $\widehat{X}$ approximates $X$. Consider a sampled block $A[k a+1,(k+1) a]$. Let $M_{k}=$ $|A[k a+1,(k+1) a] \backslash B[(k-1) a+1,(k+2) a]|$ and $N_{k}=|A[k a+1,(k+1) a] \cap B[(k-1) a+1,(k+2) a]|$. Note that $M_{k}+N_{k}=a$. When $2^{i} \leq 6400 \sqrt{a} \log \ell$, we get the exact value of $M_{k}$ and $N_{k}$ by reading the whole block. Now consider the case $2^{i}>6400 \sqrt{a} \log \ell$. Let $C_{k}$ be the number of collisions between samples from $A[k a+1,(k+1) a]$ and samples from $B[(k-1) a+1,(k+$ $2) a]$ when reading each symbol with probability $p$. We have $\mathbb{E}\left[C_{k}\right]=p^{2} N_{k}$. Consider two cases.

1. $N_{k}>\frac{0.1 \cdot 2^{i}}{2 e-1}$. By the Chernoff bound, $\operatorname{Pr}\left[\mid C_{k} / p^{2}-\right.$ $\left.N_{k} \mid \geq N_{k} \frac{0.1 \cdot 2^{i}}{N_{k}}\right] \leq 2 e^{-\left(0.1 \cdot 2^{i} / N_{k}\right)^{2} p^{2} N_{k} / 4}<\frac{1}{\ell^{2}}$.
2. $N_{k} \leq \frac{0.1 \cdot 2^{i}}{2 e-1}$. By the Chernoff bound, $\operatorname{Pr}\left[\mid C_{k} / p^{2}-\right.$ $\left.N_{k} \mid \geq N_{k} \frac{0.1 \cdot 2^{i}}{N_{k}}\right] \leq 2^{-\left(1+0.1 \cdot 2^{i} / N_{k}\right) p^{2} N_{k}}<\frac{1}{\ell^{2}}$.

Thus, with high probability, $\left|\frac{C_{k}}{p^{2}}-N_{k}\right|<0.1 \cdot 2^{i}$. Therefore, the test on line 10 passes if $M_{k} \geq 2^{i}$ and fails if $M_{k}<0.8 \cdot 2^{i}$. Let $H_{i}$ be the number of indices $k \in[\ell / a]$ such that $\mid A[k a+1,(k+1) a] \backslash B[(k-1) a+1,(k+$ $2) a] \mid \geq 2^{i}$ and $K_{i}$ be the number of indices $k \in[\ell / a]$ such that $|A[k a+1,(k+1) a] \backslash B[(k-1) a+1,(k+2) a]|>$ $0.8 \cdot 2^{i}$

By Claim 4.1, with high probability, $\frac{b}{2^{i} \log ^{3} \ell} \widehat{X}_{i}<$ $1.1 K_{i}+\frac{b}{2^{i} \log ^{2} \ell}$ and thus, $\widehat{X}<\frac{1.1 \cdot 2}{0.8} X+\frac{b}{\log \ell}$. Similarly, with high probability, $\frac{b}{2^{i} \log ^{3} \ell} \widehat{X}_{i}>0.9 H_{i}-\frac{b}{2^{i} \log ^{2} \ell}$ and thus, $\widehat{X}>0.9 X-\frac{b}{\log \ell}$.

We now show $\widehat{Y}$ approximates $Y$. Let $P_{k, t}$ be the number of characters $A[u]=B[v]$ such that $k a+1 \leq$ $u \leq(k+1) a$ and $(k-1) a+1 \leq v \leq(k+2) a$, and $|A[u-z, u-1] \Delta B[v-z, v-1]|>2 t z$ for some $z<4 a$. By Lemma 4.4 and the union bound, with probability at least $1-\frac{1}{\ell}$, all YContributingTest calls give correct answers. Each character contributing to $Y$ is picked in both $A$ and $B$ on line 11 with probability $r^{2}=O\left(\frac{\log ^{2} \ell}{2^{i}}\right)$. By Claim 4.1, with high probability, $D<1.1 \cdot P_{k, 0.4}+\frac{2^{i}}{\log \ell}$ and $D>0.9 \cdot P_{k, 0.5}-\frac{2^{i}}{\log \ell}$.

Let $P_{i}$ be the number of indices $k \in\left[\ell_{A} / a\right]$ such that $P_{k, 0.5}>2^{i}$ and $Q_{i}$ be the number of indices $k \in\left[\ell_{A} / a\right]$ such that $P_{k, 0.4}>0.8 \cdot 2^{i}$. By Claim 4.1, with high probability, $0.9 \cdot P_{i}-\frac{b}{2^{i} \log ^{2} \ell}<\frac{b}{2^{i} \log ^{3} \ell} Y_{i}<1.1 Q_{i}+$ $\frac{b}{2^{i} \log ^{2} \ell}$. Thus, $0.9 \cdot Y_{0.5}-\frac{b}{\log \ell}<\widehat{Y}<2.5 Y_{0.4}+\frac{b}{\log \ell}$.

Therefore, with high probability, if $\operatorname{ed}(A, B)<a$, $\widehat{X}+\widehat{Y}<3 a+\frac{2.5 \cdot 4}{0.4} a+\frac{2 b}{\log \ell}<b / 10$ and if $\operatorname{ed}(A, B)>b$ and $\left|\ell_{B}-\ell_{A}\right|<b / 10, \widehat{X}+\widehat{Y}>\frac{0.8 b(1-0.5)}{2} \geq b / 10$. Therefore, the algorithm answers correctly.

The expected running time of the algorithm is

$$
\begin{gathered}
\sum_{i=0}^{\log a} \frac{\ell 2^{i} \log ^{3} \ell}{a b} \tilde{O}\left(\min \left(a, \frac{a \sqrt{a}}{2^{i}}\right)+\frac{a \sqrt{a}}{2^{i}}\right)= \\
\tilde{O}\left(\frac{\ell \sqrt{a}}{b}\right) .
\end{gathered}
$$

\section{Lower bound: Proof of Theorem 1.2}

To prove Theorem 1.2, we follow the outline given in the techniques section. The lower bound $\Omega\left(\frac{d}{R}\right)$ follows directly from the folklore lower bound on testing the Hamming distance, so we concentrate on the regime $2 \sqrt{d} \leq R \leq d / 4 \alpha$.

Let $\ell=4 \alpha R$ and $r=R^{2} / d$. We define two distributions over pairs of permutations of $[\ell]$. Let $\mu_{f}$ be the distribution over pairs $(A, B)$ where $\mathrm{A}$ is a random 
permutation of $[\ell]$ and $B$ is obtained from $A$ by a cyclic rotation of $A$, moving $t_{f}$ characters at the end of $A$ to the beginning with $t_{f}$ drawn uniformly from $[\ell / 4, \ell / 2]$. Let $\mu_{c}$ be the distribution over pairs $(A, B)$ where $A$ is a random permutation and $B$ is obtained from $A$ by a cyclic rotation of $A$ by an amount $t_{c}$ drawn uniformly from $[r / 4, r / 2]$.

By an argument similar to $\mathrm{BEK}^{+} 03$, Theorem 3], there is a constant $c$ such that any deterministic algorithm that, with probability at least 5/9, distinguishes a pair $(A, B)$ drawn from $\mu_{f}$ from a pair $(A, B)$ drawn from $\mu_{c}$, must make at least $c \sqrt{r}$ queries. For completeness, below we include the sketch of the proof of the claim.

Lemma 5.1. ([ $\left.\left.\mathrm{BEK}^{+} 03\right]\right)$ For any deterministic algorithm $M$ making at most $\sqrt{r} / 5$ queries,

$$
\begin{aligned}
\operatorname{Pr}_{(A, B) \leftarrow \mu_{f}}[M(A, B)= & 1]-\operatorname{Pr}_{(A, B) \leftarrow \mu_{c}}[M(A, B)=1] \mid \\
& <1 / 9 .
\end{aligned}
$$

Proof. [Proof sketch.] Let $B$ be a cyclic rotation of $A$ by an amount $t \leq \ell / 2$. The longest common subsequence of $A$ and $B$ has length exactly $\ell-t$. Thus, when $(A, B)$ is drawn from $\mu_{f}, \operatorname{ed}(A, B) \geq t_{f} \geq \ell / 4$. When $(A, B)$ is drawn from $\mu_{c}$, ed $(A, B) \leq 2 t_{c} \leq r$. Define $R_{c}$ to be the event that the input to $M$ is drawn from $\mu_{c}$ and $M$ queries some position $i$ in $A$ and position $i+t_{c}$ in $B$ for some $i \in[\ell]$. Also define $R_{f}$ to be event that the input to $M$ is drawn from $\mu_{f}$ and $M$ queries $i$ in $A$ and $i+t_{f}$ in $B$ for some $i \in[\ell]$. When $R_{c}$ and $R_{f}$ does not happen, all queried characters are distinct and random so $M$ can not distinguish between $\mu_{c}$ and $\mu_{f}$. Thus,

$$
\begin{gathered}
\left|\underset{(A, B) \leftarrow \mu_{f}}{\operatorname{Pr}}[M(A, B)=1]-\underset{(A, B) \leftarrow \mu_{c}}{\operatorname{Pr}}[M(A, B)=1]\right| \\
\leq \max \left(\operatorname{Pr}\left[R_{c}\right], \operatorname{Pr}\left[R_{f}\right]\right) .
\end{gathered}
$$

When $M$ finds two identical characters, it can correctly distinguish between $\mu_{f}$ and $\mu_{c}$. When $M$ has not seen two identical characters in $A$ and $B$, all queried characters are random and distinct. Therefore, adaptivity does not help and we can assume $M$ makes all queries at once. After $q$ queries on $A$ and $B$, at most $(q / 2)^{2}$ shifts are checked and because $t_{c}$ and $t_{f}$ are chosen uniformly at random, we have

$$
\max \left(\operatorname{Pr}\left[R_{c}\right], \operatorname{Pr}\left[R_{f}\right]\right) \leq \max \left(\frac{(q / 2)^{2}}{r / 4}, \frac{(q / 2)^{2}}{\ell / 4}\right)<1 / 9 .
$$

This concludes the proof of Lemma 5.1.
We now proceed to proving Theorem 1.2 , Assume for contradiction that there is an algorithm $M^{\prime}$ that with probability at least $2 / 3$, takes at most $\frac{\sqrt{d}}{216 \alpha}$ queries and approximates the Ulam distance between two input strings $A, B$ up to a constant factor $\alpha$. One can construct an algorithm to distinguish $\mu_{c}$ and $\mu_{f}$ with at most $\sqrt{r} / 5$ samples as follows. Given some pair $(A, B)$ from either $\mu_{c}$ or $\mu_{f}$, construct a new pair $(P, Q)$, which consists of $d / \ell$ blocks each, where one block at a random index $k \in[d / \ell]$ is $(A, B)$ and all the others are drawn i.i.d. from $\mu_{c}$. Run $M^{\prime}$ on $(P, Q)$. If $M^{\prime}$ queries the block $(A, B)$ at least $\frac{R}{5 \sqrt{d}}$ times, then the algorithm aborts. Now, our output is "FAR" iff either

1. $M^{\prime}$ outputs "FAR", or,

2. $M^{\prime}$ takes at least $\frac{R}{5 \sqrt{d}}$ from the block $(A, B)$ (i.e., the algorithm aborts).

Clearly, our algorithm makes at most $\frac{R}{5 \sqrt{d}}$ queries to $(A, B)$.

Now, we prove the correctness of the algorithm. When $(A, B)$ is drawn from $\mu_{c}, \operatorname{ed}(P, Q) \leq d r / \ell=\frac{R}{4 \alpha}$. When $(A, B)$ is drawn from $\mu_{f}, \operatorname{ed}(P, Q) \geq \ell / 4=\alpha R$. Because $\frac{R}{4 \alpha} \cdot \alpha<R$, if aborting is ignored, $M$ should output the correct answer with probability at least $2 / 3$. If $(A, B) \in \mu_{f}$, then with probability at least $2 / 3$, the output would be "FAR" (at least by criterion 1). Now consider the case $(A, B) \in \mu_{c}$. With probability at least $2 / 3$, criterion 1 cannot happen. Let $N_{i}$ be the number of queries $M$ makes on the $i^{\text {th }}$ block. All blocks are drawn i.i.d. from $\mu_{c}$ regardless of the value of $k$ and $k$ is not revealed to $M$ so $\mathbb{E}\left[N_{i}\right]=\mathbb{E}\left[N_{i} \mid k=1\right]=\ldots=$ $\mathbb{E}\left[N_{i} \mid k=d / \ell\right] \forall i$. Thus, $\mathbb{E}\left[N_{k}\right]=\frac{\mathbb{E}\left[\sum_{i=1}^{d / \ell} N_{i}\right]}{d / \ell} \leq \frac{R}{54 \sqrt{d}}$. By the Markov inequality, with probability at least $8 / 9, N_{k} \leq \frac{R}{6 \sqrt{d}}$ so criterion 2 cannot happen, either. Therefore, with probability at least $5 / 9$, the output when $(A, B) \in \mu_{c}$ would be "CLOSE".

The resulting algorithm distinguishes $\mu_{c}$ from $\mu_{f}$ which contradicts Lemma 5.1

The claim for edit distance on binary strings follows immediately using Theorem 1.2 of AK07.

\section{References}

[ACCL07] Nir Ailon, Bernard Chazelle, Seshadhri Comandur, and Ding Liu. Estimating the distance to a monotone function. Random Structures and Algorithms, 31:371-383, 2007. Previously appeared in RANDOM'04.

[AIK09] Alexandr Andoni, Piotr Indyk, and Robert Krauthgamer. Overcoming the $\ell_{1}$ non-embeddability barrier: Algorithms for product metrics. In Proceedings of the ACM-SIAM Symposium on Discrete Algorithms (SODA), pages 865-874, 2009. 
[AJKS02] Miklós Ajtai, T. S. Jayram, Ravi Kumar, and D. Sivakumar. Approximate counting of inversions in a data stream. In Proceedings of the Symposium on Theory of Computing (STOC), pages 370-379, 2002.

[AK07] Alexandr Andoni and Robert Krauthgamer. The computational hardness of estimating edit distance. In Proceedings of the Symposium on Foundations of Computer Science (FOCS), pages 724-734, 2007. Accepted to SIAM Journal on Computing (FOCS'07 special issue).

[AK08] Alexandr Andoni and Robert Krauthgamer. The smoothed complexity of edit distance. In Proceedings of International Colloquium on Automata, Languages and Programming (ICALP), pages 357-369, 2008.

[AO09] Alexandr Andoni and Krzysztof Onak. Approximating edit distance in near-linear time. In Proceedings of the Symposium on Theory of Computing (STOC), pages 199-204, 2009.

$\left[\mathrm{BEK}^{+}\right.$03] Tuğkan Batu, Funda Ergün, Joe Kilian, Avner Magen, Sofya Raskhodnikova, Ronitt Rubinfeld, and Rahul Sami. A sublinear algorithm for weakly approximating edit distance. In Proceedings of the Symposium on Theory of Computing (STOC), pages 316-324, 2003.

[GJKK07] Parikshit Gopalan, T. S. Jayram, Robert Krauthgamer, and Ravi Kumar. Estimating the sortedness of a data stream. In Proceedings of the ACMSIAM Symposium on Discrete Algorithms (SODA), pages 318-327, 2007.

[KR06] Robert Krauthgamer and Yuval Rabani. Improved lower bounds for embeddings into $L_{1}$. In Proceedings of the ACM-SIAM Symposium on Discrete Algorithms (SODA), pages 1010-1017, 2006.

[Mar95] John I. Marden. Analyzing and Modeling Rank Data. Monographs on Statistics and Applied Probability 64. CRC Press, 1995.

[MR95] Rajeev Motwani and Prabhakar Raghavan. Randomized Algorithms. Cambridge University Press, 1995. 